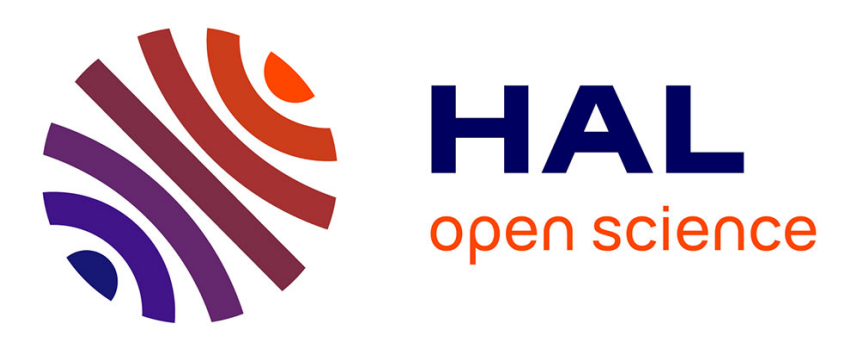

\title{
Influence de la qualité et de la quantité de l'alimentation sur les fluctuations des populations de Lymantria dispar L. (Lep. Lymantriidae) en forêt de la Mamora (Maroc)
} Alain Fraval

\section{- To cite this version:}

Alain Fraval. Influence de la qualité et de la quantité de l'alimentation sur les fluctuations des populations de Lymantria dispar L. (Lep. Lymantriidae) en forêt de la Mamora (Maroc). Agronomie, 1984, 4 (9), pp.819-828. hal-00884702

\section{HAL Id: hal-00884702 https://hal.science/hal-00884702}

Submitted on 1 Jan 1984

HAL is a multi-disciplinary open access archive for the deposit and dissemination of scientific research documents, whether they are published or not. The documents may come from teaching and research institutions in France or abroad, or from public or private research centers.
L'archive ouverte pluridisciplinaire HAL, est destinée au dépôt et à la diffusion de documents scientifiques de niveau recherche, publiés ou non, émanant des établissements d'enseignement et de recherche français ou étrangers, des laboratoires publics ou privés. 


\section{Influence de la qualité et de la quantité de l'ali- mentation sur les fluctuations des populations de Lymantria dispar L. (Lep. Lymantriidae) en forêt de la Mamora (Maroc)}

Alain FRAVAL

Institut Agronomique et Vétérinaire Hassan II, Département de Zoologie, B.P. 6202, Rabat-Instituts Maroc

Lymantria dispar $\mathrm{L}$. est un ravageur cyclique des suberaies de la bordure atlantique du Maroc. L'abondance du feuillage qualitativement satisfaisant offert par l'arbre-hôte, Quercus suber L., varie largement en fonction de l'année et de l'intensité du débourrement.

Différentes situations trophiques ont été observées depuis 1976 sur un transect fixe de $7 \mathrm{~km}$ de long installé en forêt de la Mamora. Leurs répercussions sur les populations du ravageur sont décrites et examinées à la lumière des résultats d'élevages au laboratoire conduits par HÉrard (1984).

Il est apparu en outre que $Q$. suber ne débourre pas ou bien débourre très tardivement l'année suivant sa défoliation par L. dispar.

$\mathrm{La}$ régulation des populations est assurée, dans un premier temps, par la famine consécutive à la défoliation complète puis, à la génération suivante, par le défaut qualitatif de nourriture dû à la réaction de $Q$. suber. Ce mécanisme n'explique pas seul les fluctuations de populations du ravageur enregistrées à l'échelle de la forêt.

Mots clés additionnels : Quercus suber $L$., relations plante-insecte, alimentation, discordance phénologique.

Population fluctuation in the gypsy moth, Lymantria dispar L. (Lep. : Lymantriidae), in the Mamora forest (Morocco), governed by food quality and quantity.

Lymantria dispar L. occurs in Morocco in all oak forests. Along the Atlantic coast it periodically causes heavy defoliation of the cork-oak, Quercus suber L. A preliminary study made by DE LEPINEY (1930) showed that the main factors responsible for the decline of an infestation were natural enemies, shortage of food after complete defoliation of the cork-oak, Quercus suber L. A preliminary study made by DE LEPINEY (1930) showed that the main factors responsible for the decline of an infestation were natural enemies, shortage of food after complete defoliation, and diachronism between the formation of new foliage and the hatching of eggs. Recent investigations are reported here. Data were collected from 1976 on density estimations of the insect pest, description of the phenology of the tree and checking of defoliation along a transect in the Mamora forest (near Kenitra). The effects of various trophic conditions are described according to the results of previous laboratory experiments (HérARD, 1984). The behaviour of the foliage of the cork-oak (fig. 1) depends on the occurrence of complete defoliation in the previous year. Trees that are defoliated show new foliage after a few weeks, and bud break does not occur next spring; the old leaves persist at high density. The gypsy moth populations are regulated by the following mechanism : shortage of food kills many caterpillars and survivors have a reduced fecundity (fig. 2). Larvae of the next generation eat old and non preferred foliage (delayed reaction of the tree) ; many die, fecundity and fertility are affected and the population collapses. A theoretical scheme of population fluctuation has been drawn up on the basis of this regulatory mechanism, in accordance with the changes registered along the transect. This mechanism seems to be specific to some Moroccan corkoak forests. Other factors are suspected to play a major role in the population dynamics of the gypsy moth, such as climate and physical and chemical properties of the leaves. Mortality due to natural enemies was inconsistent.

Additional key words : Quercus suber $L$., insect-plant relationships, alimentation, phenological disruption. 


\section{INTRODUCTION}

Depuis le début du siècle, Lymantria dispar L., le bombyx disparate, se manifeste de façon grossièrement cyclique par des défoliations spectaculaires dans certaines des suberaies de la bordure atlantique du Maroc (Fraval \& HÉRARD, 1975). Ces forêts sont des peuplements quasi purs de chêne-liège, Quercus suber L.

La vaste forêt de la Mamora (environ 130000 ha en 1920,65000 ha de $Q$. suber actuellement), proche de Rabat, a fait très tôt l'objet d'observations entomologiques concernant les défoliations du chêne et $L$. dispar y a été signalé dès 1919 (SCHINDLER, 1921). Ses ravages inquiétèrent les Services Forestiers, nouvellement créés, qui se préoccupaient de restaurer la forêt de la Mamora, en très mauvais état. L'étude de l'insecte fut confiée à DE LÉPINEY. On doit à cet auteur la $1^{\text {re }}$ tentative d'explication des pullulations observées (DE LÉPINEY, 1930).

A partir de 1972, le ravageur a fait l'objet d'une nouvelle étude qui a débuté par un inventaire des ennemis naturels (FRAVAL \& HÉRARD, 1975; HÉRARD \& FR.AVAL, 1980). Les observations alors réalisées nous ont conduit à considérer les perturbations de l'alimentation (discordance phénologique et épuisement du feuillage) comme prééminentes parmi les facteurs de la régulation des populations de l'insecte, les ennemis naturels et les aléas climatiques étant jugés d'importance mineure (FRAVAL et al., 1975).

Pour préciser l'effet des conditions trophiques sur $L$. dispar, 2 séries de travaux ont été entreprises, successivement :

- les conséquences sur les individus de défauts qualitatifs et quantitatifs de nourriture ont été étudiées au laboratoire par HÉRARD (1984) ;

- l'évolution des effectifs de $L$. dispar, celle du feuillage du chêne liège et leurs interactions ont été décrites au moyen d'estimations et mesures régulières réalisées le long d'un transect implanté en forêt de la Mamora.

Une $1^{\text {re }}$ analyse du rôle du facteur trophique dans la dynamique des populations de $L$. dispar a été présentée par ailleurs (FRAVAL, 1979).

Dans la présente note, nous examinerons les conséquences sur l'insecte de 4 situations trophiques simples (définies d'après nos $1^{\text {res }}$ observations), ceci à partir des phénomènes observés sur le transect et en nous référant aux résultats des expériences de HÉRARD (1984). La mise en évidence de la réaction du chêneliège à la défoliation nous permettra de proposer un schéma explicatif des fluctuations locales des effectifs de $L$. dispar, que nous confronterons aux évolutions observées.

\section{LYMANTRIA DISPAR}

\section{SES RELATIONS AVEC SES PLANTES-HÔTES}

\section{A. Généralités}

L'espèce est monovoltine. La femelle pond, en juinjuillet, de 200 à 600 œufs groupés en une ooplaque. L'œuf, au bout de 2 à 3 semaines de développement embryonnaire, contient une chenille formée qui passe l'été puis l'hiver en diapause (" estivo-hivernation »). La chenille se développe au printemps sur les jeunes feuilles d'une grande diversité de plantes. La nymphose dure 2 semaines environ. Les adultes ne se nourrissent pas.

La répartition géographique est très vaste, depuis le Japon jusqu'aux U.S.A. Les déplacements sont surtout passifs, les 2 vecteurs principaux étant l'homme et le vent. Les papillons femelles ne volent pas, en général.

Les limites théoriques de la polyphagie sont très étendues (SCHEDL, 1936). Selon l'habitat, cette polyphagie s'exprime plus ou moins, en fonction de la diversité offerte. Dans les peuplements forestiers mélangés (très fréquents en Amérique du Nord), certaines associations d'essences sont plus favorables que d'autres à $L$. dispar et leur répartition contribue à déterminer les zones sensibles; ces dernières sont caractérisées en outre par la présence d'abris (BESS et al., 1947 ; BARBOSA, 1978).

Sur une espèce végétale capable d'assurer le développement complet de l'insecte, la survie, la croissance et la reproduction dépendent du lieu d'alimentation (POTTS, 1938), de l'âge des feuilles (RIDET, 1972, Hough \& PIMENTEL, 1978), de l'intégrité des feuilles voisines (WALLNER \& WALTON, 1979). L'évolution de L. dispar est également fonction de l'état physiologique de l'arbre donc de l'âge de celui-ci, du mode de conduite de la forêt, des conditions pédoclimatiques, des actions de l'homme. Le développement de l'insecte est plus rapide et sa survie plus probable sur du feuillage d'arbres mal venants, chétifs, abîmés (VON RUDNEV, 1963 ; PATOČKA, 1973).

On connaît mal celles des caractéristiques physiques et chimiques des feuilles qui conditionnent leur qualité alimentaire pour $L$. dispar. FEENY (1970) constate, au cours du printemps, la baisse de la qualité de la feuille de $Q$. robur L. vis-à-vis de Operophtera brumata L. (Lep. : Geometridae) ; il l'attribue à l'épaississement du limbe et à la diminution de l'azote disponible, liée en partie à l'augmentation de la concentration en tannins.

Les effets d'un jeûne limité et localisé dans le temps sont décrits par LEONARD (1966) à partir d'élevages sur milieu artificiel : l'activité de déambulation est accrue et le nombre de stades larvaires est modifié, augmenté si la privation de nourriture est appliquée au $1^{\text {er }}$ stade, réduit si elle est appliquée au début des stades ultérieurs.

Ce même auteur (LEONARD, 1970) considère que les effectifs des populations de $L$. dispar sont autorégulés par l'intervention de modifications qualitatives d'une partie des individus, induites par des changements dans l'alimentation résultant de l'accroissement de la densité. La survie de ces individus est assurée par l'émigration passive (par le vent), pour laquelle leurs capacités sont accrues (les jeunes chenilles issues d'œufs petits sont plus actives et le $1^{\text {er }}$ stade est prolongé) ; les individus demeurant sur place sont désavantagés (développement plus lent) et condamnés à terme.

Pour CAMPBELL \& SLOAN (1978), qui s'opposent à nombre de conclusions de LEONARD, les populations de $L$. dispar évoluent selon 2 régimes distincts : l'un 
où la densité reste perpétuellement faible, l'autre où elle oscille. Dans ce $2^{\mathrm{e}}$ cas, une régulation par les effets de la surpopulation intervient pour des densités très inférieures à celles correspondant à la culmination et à la rétrogradation brutale par épizootie et famine : la femelle pond moins d'œufs et le développement larvaire est accéléré ce qui, en cas d'épuisement du feuillage (phénomène peu fréquent), assure la survie d'un nombre suffisant d'individus pour la perpétuation de l'infestation sur place.

La privation de nourriture consécutive à la défoliation totale des plantes-hôtes n'est reconnue que rarement comme un facteur décisif du devenir de la population ; citons les observations de FORBUSH \& FERNALD (1896) au début de l'expansion de l'espèce aux U.S.A., celles de divers auteurs, au début du siècle, en Crimée et en Dalmatie, citées par SCHEDL (1936), celles de DOANE (1968) évaluant les conséquences d'un traitement. Seul, à notre connaissance, DE LEPINEY (1930) y voit un facteur régulier.

\section{B. Lymantria dispar au Maroc}

Au Maroc, la répartition géographique de $L$. dispar se superpose à celle des chênes : Quercus suber L., $Q$. ilex L. (chêne-vert), $Q$. mirbeckii Dur. (chêne-zéen) (FrAVAl et al., 1975) ; les Quercus spp. se montrent seuls capables en nature d'assurer le développement complet de l'insecte.

Les $L$. dispar de la suberaie atlantique se distinguent de ceux de la yeuseraie par le comportement de ponte et les capacités d'allotrophie vis-à-vis du peuplier (HÉRARD, 1984). Les ennemis naturels sont peu diversifiés (HÉRARD \& FRAVAL, 1980), observation qui corrobore l'opinion de GOLDSCHMIDT (1934) selon laquelle $L$. dispar aurait été introduit récemment au Maroc, par l'homme. Une particularité remarquable des $L$. dispar de suberaie est l'étalement des éclosions sur plus d'un mois.

Pour DE LEPINEY (1930), la dynamique des populations du ravageur est régie par les ennemis naturels, par la famine consécutive aux défoliations totales et par la discordance phénologique entre l'éclosion des chenilles et le débourrement ; dans ce cas, l'insecte est contraint de s'alimenter sur le feuillage formé l'année précédente, dit feuillage « ancien ».

En Mamora, d'après nos observations, L. dispar est le seul insecte phyllophage causant des dégâts notables au chêne-liège. La dernière période de pullulation y a débuté en 1971 et s'est achevée en 1982.

\section{DISPOSITIFS EXPÉRIMENTAUX}

\section{A. Les essais au laboratoire (HÉRARD, 1984)}

Ils ont été conçus pour décrire les effets immédiats (sur la survie et la croissance) et différés (sur le nombre et la vitalité des descendants) de séquences d'ali- mentation établies a priori, reproduisant des situations trophiques observées en nature ; ces séquences font se succéder des périodes d'alimentation sur feuillage jeune, sur feuillage ancien et des périodes de jeûne. Les mesures portent notamment sur la quantité de feuillage consommé, le poids des fèces, la survie et l'accroissement pondéral des chenilles, la fertilité des femelles, la survie des œufs. Les chenilles sont élevées sur du feuillage frais excisé de $Q$. suber. Une climatisation reproduit approximativement les conditions moyennes de la Mamora au printemps (FRAVAL \& HÉRARD, 1975).

\section{B. Le transect et les techniques de dénombrement}

Le transect est constitué d'une soixantaine de stations espacées de $100 \mathrm{~m}$ et comportant chacune 10 arbres. Sa longueur totale est de $7 \mathrm{~km}$. Situé au nord du canton B de la Mamora, non loin de Kénitra, il traverse des peuplements de $Q$. suber, avec quelques rares Pirus mamorensis Trabut ; le sous-bois est constitué notamment de genêt Teline linifolia $\mathrm{L}$. et de doum, Chamaerops humilis L. Les caractéristiques du transect ont été déterminées de façon à enregistrer les phénomènes en relation avec l'évolution du feuillage du chêne, les plus variables d'un endroit à l'autre, et à disposer simultanément de situations variées; on a tenu compte des connaissances acquises sur la répartition de l'insecte (FRAVAL et al., 1978). Les contraintes de temps et de personnel, les difficultés de repérage en forêt à sous-bois dense, ainsi que les possibilités graphiques offertes ont conduit à choisir un dispositif linéaire.

Les relevés sont effectués chaque semaine (voire avec une fréquence moindre) durant la vie des chenilles, des chrysalides et des imagos sur tous les arbres du dispositif, par une équipe d'observateurs et en une journée (FRAVAL et al., 1980).

Pour chaque arbre, les effectifs de pontes, de chenilles, de femelles en train de pondre sont dénombrés. On note simultanément la proportion de feuillage ancien, de feuillage nouveau en cours de croissance, de feuillage nouveau ayant achevé sa croissance, de feuillage "supplémentaire » en train de pousser, de feuillage «supplémentaire » à croissance achevée, ainsi que l'importance des dégâts de $L$. dispar par catégorie de feuillage, avec indication de la répartition des niveaux d'attaque ; on note également la présence de fleurs.

L'impact des ennemis naturels est estimé à l'aide de prélèvements suivis d'élevages (de pontes, chenilles et chrysalides) et par observations à vue.

Des observations complémentaires sont apportées par d'autres travaux de terrain, réalisés également dans la partie occidentale de la forêt de la Mamora. Ces travaux ont eu pour but d'expérimenter des moyens de lutte (FRAVAL et al., 1977a et $b$; FRAVAL et al., 1981), de suivre comparativement l'évolution de $L$. dispar en différents placeaux de la suberaie (FRAVAL \& MAZIH, 1980; FRAVAL \& BENLAHBOUBJAZOULI, 1984) et de déterminer les risques de défoliation (FrAVAL \& LHALOUI, 1980). L'articulation des différents travaux consacrés à $L$. dispar a été expliquée par ailleurs (FRAVAL, 1983). 


\section{RÉSULTATS}

\section{A. Effets sur $L$. dispar de 4 types de situations trophiques}

Par situation trophique nous entendons l'état de la nourriture et son évolution au cours de la vie larvaire de l'insecte. Ces situations sont observées dans la nature, sur le transect notamment, ou créées au laboratoire.

\section{Situation optimale}

Nous dénommons ainsi la situation qui garantit la mortalité la plus faible, la croissance la plus rapide et la plus forte, les meilleures fécondités et fertilités, la survie du plus grand nombre de chenilles en diapause dans l'œuf. En élevage elle est obtenue sur du feuillage fraîchement formé; le développement larvaire dure $48 \mathrm{j}$, le développement nymphal $14 \mathrm{j}$ (mâles) ou $12 \mathrm{j}$ (femelles) ; la mortalité totale est de 6 p. 100 ; chaque femelle pond 450 œufs environ, dont 95 p. 100 éclosent (HÉRARD, 1984).

En forêt, cette situation n'a été rencontrée qu'au cours du cycle 1975-1976, correspondant à nos $1^{\text {res }}$ observations : par suite d'une très importante mortalité des chenilles en diapause dans l'œuf, très peu de chenilles ont éclos des pontes déposées par les femelles de la génération précédente (proportion d'œufs éclos < 1 p. 100). A ces chenilles, les chêneslièges dont le débourrement a été précoce, ont offert un feuillage favorable, pendant toute la saison; la défoliation n'a pas dépassé 10 à 15 p. 100 de la masse du feuillage. Les chrysalides étaient grosses et le taux sexuel avoisinait l'unité. Les pontes, déposées en juillet 1976, étaient relativement peu nombreuses (4 à 35 par arbre) et de grande taille; elles contenaient au printemps suivant une forte proportion d'œufs viables (30 à 50 p. 100).

Dans cette situation trophique, les jeunes chenilles quittent la ponte au bout de 1 à $2 \mathrm{j}$, grimpent et s'arrêtent pour se nourrir sur les feuilles tendres; la chenille au $1^{\text {er }}$ stade décape le limbe puis elle le découpe ( $2^{e}$ stade), en «Randfresser 》 le plus souvent. Les rares feuilles anciennes ou desséchées, voire les feuilles plus âgées, sont préférées pour le repos diurne des stades les plus âgés et pour la mue. Là s'observent les rares individus parasités par Apanteles spp. et Meteorus pulchricornis Wesmael (Hym. : Braconidae).

\section{Situation de déficience quantitative}

Une telle situation est observée lorsque les chenilles ont épuisé tout le feuillage disponible, ce qui arrive au-delà du $4^{e}$ stade larvaire pour de très fortes densités de population. Elle correspond à des séquences de conditions alimentaires où la pénurie absolue est précédée d'une pénurie relative, la compétition ayant pour effet que chaque individu est sous-alimenté ; dans le cas d'un feuillage mixte (nouveau et ancien), le feuillage nouveau est vite épuisé et la phase de jeûne strict est précédée d'une phase d'alimentation qualitativement déficiente (cf. situation 3 , ci-après).
En élevage, le jeûne ralentit puis annule la croissance ; la mortalité est très importante ; les papillons femelles pondent très peu d'œufs ; ces œufs sont viables (HÉRARD, 1984).

En forêt, de telles situations ont été observées sur une partie du transect pour le cycle 1976-1977, sur tout le transect pour le cycle 1977-1978, sur une autre partie du transect pour le cycle 1979-1980 (fig. 1). Une forte mortalité a été alors observée : les chenilles s'amoncellent au pied des arbres ou restent suspendues ou accrochées aux aspérités du liège. Les chrysalides sont de petite taille et en majorité de sexe mâle ; les chenilles mâles en effet, moins exigeantes et au développement plus rapide, ont plus de chances de parvenir à se chrysalider. La réduction de poids des chrysalides femelles consécutive à l'intensité et à la précocité de la défoliation (fig. 2) a aussi pour conséquence un abaissement de la fécondité. Ces phénomènes sont plus ou moins marqués selon la période à laquelle survient la défoliation ; une défoliation relativement tardive laisse plus de survivants avec des potentialités moins diminuées.

Des observations en placeaux, plus précises, mettant en relation les mortalités enregistrées avec l'évolution de la population dans le temps (Fraval \& MAZIH, 1980), confirment ces observations. Elles montrent que les effectifs des populations étudiées s'effondrent au moment de la défoliation complète des arbres. Seule survit une petite cohorte d'individus précoces.

En situation de disette, on remarque en outre des modifications importantes du comportement alimentaire :

- l'activité diurne des chenilles augmente; elles explorent avidement le milieu, arbres et sous-bois. Cette quête peut conduire à l'extension de la zone défeuillée de 100 à $200 \mathrm{~m}$, comme ceci a été observé en 1980 vers les stations 126-127 du transect ;

- d'autres végétaux que le chêne-liège sont consommés. Dans le cas d'un feuillage mixte (ancien et nouveau), l'exploration commence dès la disparition du feuillage jeune et les chenilles attaquent alors, simultanément, le feuillage ancien du chêne-liège, le doum et le poirier sauvage (observations faites en 1978 et en 1980). En bordure de forêt, les chenilles envahissent arbres fruitiers et cultures.

Les chenilles, jusqu'au $4^{\mathrm{e}}$ stade, attaquent les feuilles anciennes en les décapant; au-delà, elles pratiquent des trous dans le limbe et des découpures marginales.

\section{Situation de déficience qualitative}

Cette situation correspond à une alimentation totale ou partielle sur le feuillage ancien du chêne-liège lorsque l'arbre débourre avec retard (discordance phénologique) ou quand le feuillage nouveau a été épuisé.

Au laboratoire, dans tous les cas observés, le feuillage nouveau est préféré. Le choix s'effectue au contact, après des mouvements au hasard. La feuille jeune est consommée, la feuille ancienne incite la chenille affamée à poursuivre son chemin.

Les chenilles au $1^{\text {er }}$ stade, contraintes en laboratoire de s'alimenter plus de $7 \mathrm{j}$ sur du feuillage ancien (ce 

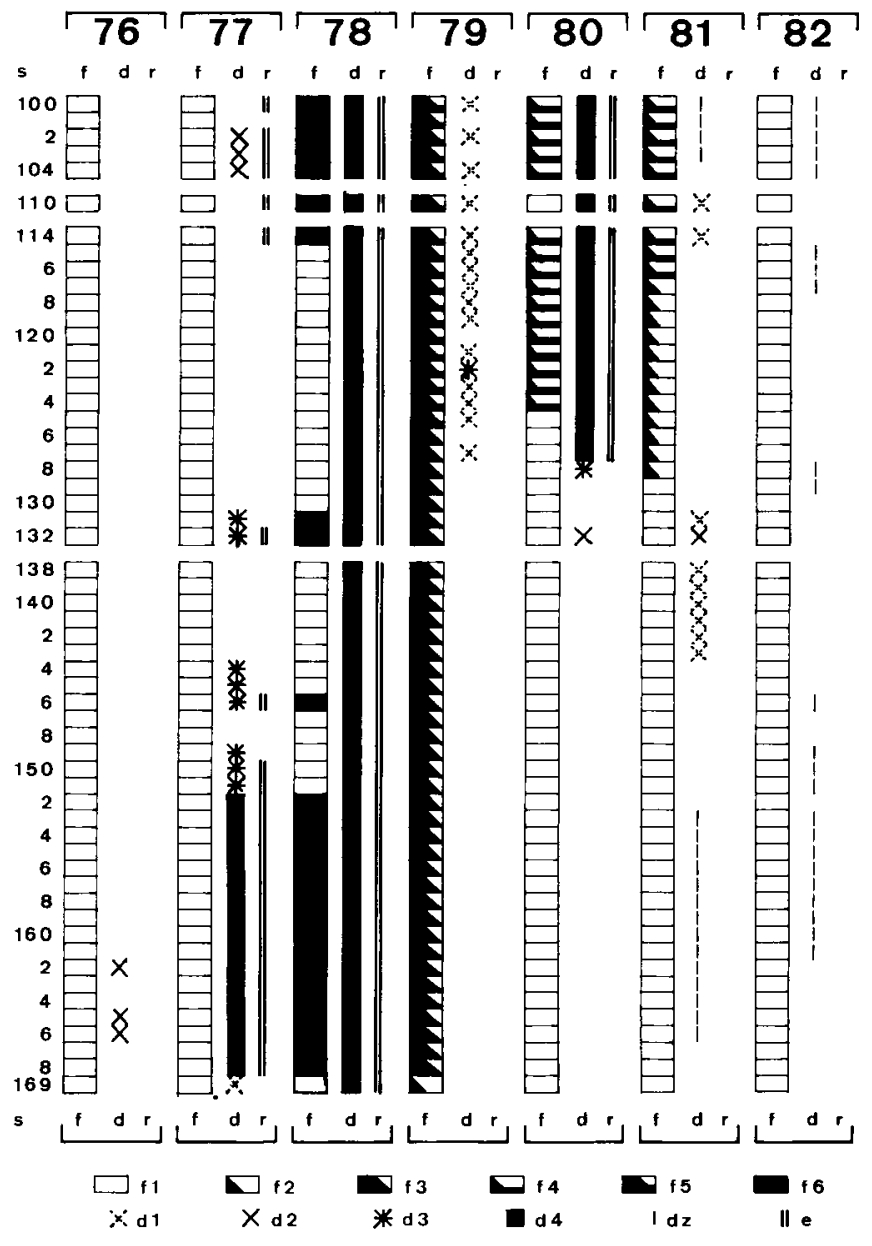

Figure 1

Principaux traits de l'évolution du feuillage des chênes-lièges observés de 1976 à 1982, le long du transect.

$\mathbf{s}$ : stations du transect, $\mathbf{f}:$ feuillage de printemps, à l'époque de la vie larvaire de Lymantria dispar ; $\mathbf{f} 1$ : débourrement précoce, feuillage favorable; $\mathbf{f} \mathbf{2}$ : débourrement complet et tardif ; $\mathbf{f 3}:$ id., très tardif ; $\mathbf{f 4}$ : débourrement partiel et tardif ; $\mathbf{5}$ : id., très tardif ; $\mathbf{f 6}$ : pas de débourrement. $\mathbf{d}$ : défoliation; $\mathbf{d 1}:$ faible; $\mathbf{d} 2$ : moyenne; $\mathbf{d 3}$ : forte; $\mathbf{d 4}$ : complète; $\mathbf{d z}:$ absence de toute chenille de $\mathrm{L}$. dispar. e : renouvellement du feuillage au début de l'été.

Main features of the development of cork-oak foliage as recorded from 1976 to 1982 along the transect.

s: plots of the transect. f : spring foliage at gypsy moth larva time; $\mathbf{f 1}$ : early flushing, foliage of the preferred type; $\mathbf{2}$ : complete but late flushing; $\mathbf{f 3}:$ id., very late ; $\mathbf{4} 4$ : partial and late flushing; $\mathbf{f 5}$ : id., very late; $\mathbf{f 6}$ : no flushing. $\mathbf{d}$ : defoliation; $\mathbf{d 1}$ : weak ; d2 : medium; $\mathbf{d 3}$ : severe ; d4 : complete; dz: no gypsy moth caterpillars. : cork-oak exhibits new foliage in early summer.

qui reproduit une situation de discordance phénologique) subissent une mortalité importante et la mue est retardée ; les survivantes ne pâtissent de cette alimentation sur feuillage ancien que si elle se prolonge audelà d'une vingtaine de jours : la fécondité est alors réduite et la survie embryonnaire moins bonne (HÉRARD, 1984).

Sur le terrain, une situation aussi nette n'est pas apparue. En effet, même si tous les arbres conservent leur feuillage ancien, beaucoup produisent des repousses très localisées à l'extrémité de certains rameaux où les chenilles se concentrent. Certains arbres renouvellent leur feuillage : ce sont des sujets abîmés (prélèvements des riverains pour la ramée).
Sur les arbres conservant leur feuillage ancien, de très nombreuses jeunes chenilles se suspendent à un long fil de soie ; le vent les entraîne. Les conditions et les conséquences de cette émigration ne nous sont pas connues.

\section{Situation la plus défavorable}

Elle combine séquentiellement tous les déficits alimentaires précédents : les arbres débourrent partiellement et en retard par rapport aux éclosions, les chenilles sont très nombreuses. Le feuillage offert est successivement : du feuillage ancien peu consommé, une petite quantité de feuillage nouveau très vite épuisée, puis à nouveau du feuillage ancien, jusqu'à épuisement.

Cette séquence, en élevage, se traduit par une forte mortalité des chenilles en " estivo-hibernation " dans le chorion (HÉRARD, 1984).

Le long du transect, nous avons observé une telle situation en 1978 de la station 152 à la station 168 et, en 1980, de la station 100 à la station 127 (fig. 1). Les conséquences ont été la quasi-disparition de $L$. dispar de ces zones.

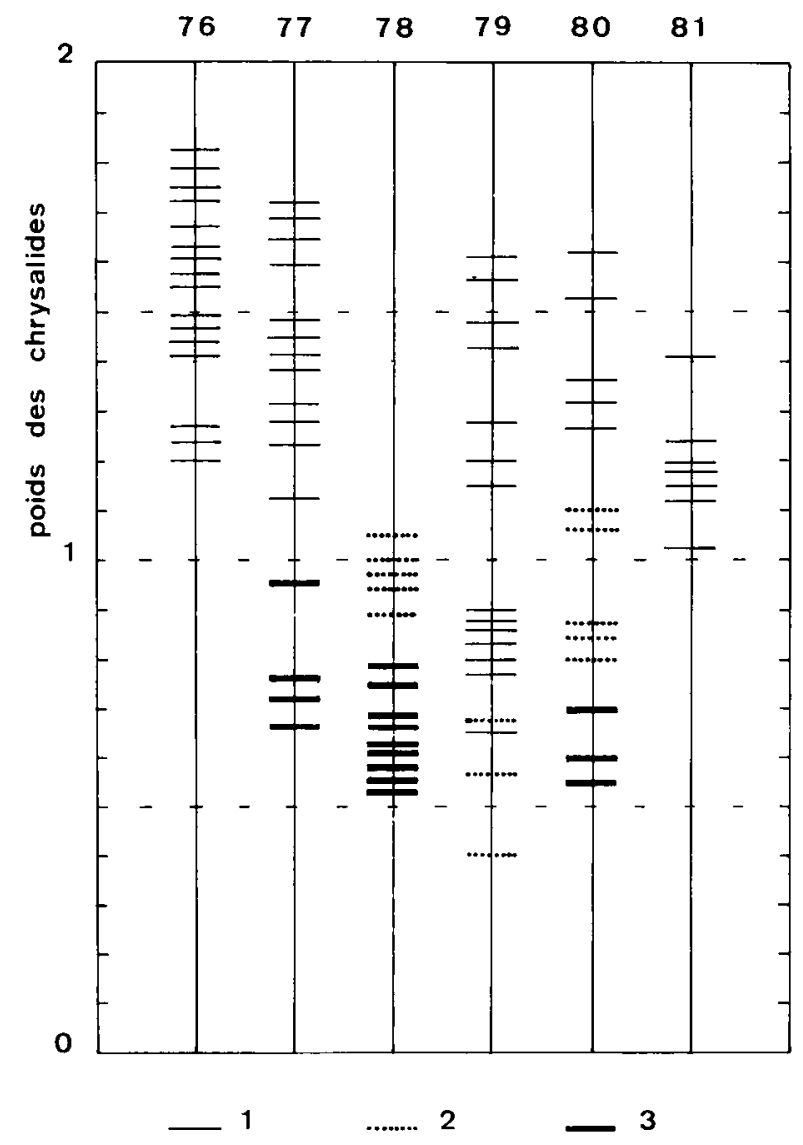

Figure 2

Poids frais (en g) des chrysalides femelles de Lymantria dispar sur différentes stations du transect; années 1976 à 1981.

1 : pas de défoliation; 2 : défoliations tardives (1978 et 1980) ou incomplètes (1979) ; 3 : défoliations totales.

Weight $(g)$ of live female gypsy moth pupae on different plots of the transect; years 1976 to 1981.

1 : no defoliation ; 2 : defoliation occurred late (1978 and 1980) or was incomplete (1979) ; 3 : complete defoliation. 
Ces expériences et observations montrent la très grande dépendance de $L$. dispar vis-à-vis des conditions trophiques. Les meilleures conditions de développement sont réalisées pour des populations peu denses en présence de feuillage nouveau. Les défauts qualitatifs et quantitatifs altèrent la croissance des chenilles, la fécondité des femelles ainsi que la survie des œufs de la génération suivante. Ceci est un mécanisme régulateur, par l'adaptation des effectifs aux ressources offertes.

\section{B. Mise en évidence d'une réaction phénologique du chêne-liège à la défoliation}

Le chêne-liège, arbre à feuilles persistantes, renouvelle généralement ses feuilles au printemps. BOUDY (1950) note que les $Q$. suber de la Mamora sont caractérisés par une chute massive des feuilles au printemps, suivie du débourrement. Pour Camus (1938), les feuilles tombent tard en saison et le débourrement est partiel.

Les données des relevés du transect, en $1^{\text {re }}$ analyse, montrent que le débourrement peut s'effectuer de façons très diverses : i广 peut être synchrone sur tous les arbres d'une surface de quelques ha et du type décrit par BOUDY (1950), comme il peut affecter seulement la moitié d'un sujet.

A partir d'un échantillon de 89 arbres du transect, observés pendant 3 ans, nous sommes parvenus à définir 6 catégories principales (FRAVAL, 1979). Un programme de tri informatique (CHEMAOU EL FIHRI, comm. pers. 1982) a permis le traitement des données de 7 années pour tous les arbres du transect ; il a conduit à une multiplication considérable du nombre des cas. Chaque arbre possède un comportement propre. Il se dégage cependant les faits suivants :

- la défoliation totale du chêne-liège par $L$. dispar (en mai) est presque toujours suivie, au bout de 3 à 4 semaines, d'une refeuillaison complète (en juin). Certains arbres, défeuillés en 1975, ne se sont refeuillés qu'au printemps suivant ; ce cas ne s'est jamais représenté (fig. 1) ;

- la refeuillaison d'été peut se manifester chez des arbres peu ou pas défeuillés, ainsi que nous l'avons observé en 1977 sur une partie du transect où les arbres sont plus jeunes ; une telle situation n'a pas été observée depuis ;

- l'année suivant la défoliation-refeuillaison, le débourrement ne se fait pas (sauf sur quelques rameaux à l'extrémité des branches), cas observé en 1978 sur la partie du transect défeuillée en 1977 (fig. 1), ou bien il se fait, plus ou moins complètement, mais il est très tardif, créant une situation de discordance phénologique. Cette dernière situation a été observée en 1979, 1980 et 1981 (fig. 1). Le feuillage ancien persiste alors au-delà de la date normale de chute des feuilles, ce qui résulte du fait que les feuilles de $Q$. suber ne tombent que lorsqu'elles ont atteint un certain degré de sénescence (AllAOUI, comm. pers.) ;

- 2 années après une défoliation complète, le chêne-liège débourre précocement (avant ou pendant l'éclosion de $L$. dispar) et complètement, ce que nous avons observé en 1980 dans la zone défeuillée en 1977 et 1978, en 1982 dans la zone défeuillée en 1980. Ce type de débourrement est observé les années suivantes (fig. 1), jusqu'en 1983.

Ainsi il apparaît que le chêne-liège renouvelle son feuillage chaque printemps sauf s'il a été défeuillé l'année précédente, auquel cas le débourrement est annulé ou retardé ce qui ménage des conditions trophiques défavorables pour $L$. dispar.

Cette réaction du chêne n'interdit pas 2 défoliations totales successives (fait constaté en 1977 et 1978 sur un tronçon du transect long de 1,7 km) mais assure la rétrogradation (constatée en 1979 et 1981) car les rares survivants à la famine sont confrontés à une nourriture qualitativement déficiente.

\section{Rôle du facteur trophique dans le déroulement des gradations locales de $L$. dispar en forêt de la Mamora}

\section{Schéma type d'une gradation locale}

A partir des faits observés et des relations de causes à effets mises en évidence, nous pouvons établir un schéma qui correspond à une séquence-type de l'évolution simultanée des populations de $L$. dispar et du feuillage de $Q$. suber au cours d'une gradation (fig. 3 ).

Les 2 premières années, les chenilles peu nombreuses disposent de feuillage favorable en quantité suffisante, d'où, entre les $2^{\mathrm{e}}$ et $3^{\mathrm{e}}$ générations, un très fort accroissement des effectifs: l'effectif de chenilles outrepasse alors la capacité du milieu et les arbres sont défeuillés. Les survivantes pâtissent des effets d'une mauvaise alimentation et la $4^{\mathrm{e}}$ génération, très peu nombreuse, se trouve en présence d'un feuillage défavorable ; ses effectifs sont encore réduits par les ennemis naturels.

Ce schéma a été effectivement observé dans la nature, dans 13 stations du transect (de 138 à 151).

\section{Cas particuliers}

- Deux défoliations successives peuvent se produire, comme ce fut le cas pour 17 autres stations (152 à 168), en 1977 et 1978 .

La défoliation tardive de 1977 a laissé un grand nombre de survivantes n'ayant subi qu'un défaut quantitatif de nourriture. La génération suivante fut assez nombreuse pour consommer tout le feuillage (ancien) des arbres mais subit en conséquence des pertes telles que $L$. dispar a apparemment disparu.

- Dans 13 autres stations (115 à 127), à une défoliation complète (en 1978) a succédé une période d'accalmie relative puis une nouvelle attaque (en 1980), suivie de la quasi-disparition du ravageur.

Au départ du cycle 1977-1978, les effectifs, dans cette zone étaient faibles, voire nuls (FRAVAL et al., 1980) ; la défoliation complète des arbres a été provoquée par un nombre relativement peu élevé de chenilles ayant à leur disposition du feuillage favorable (stations 115 à 127). L'année suivante (en 1979), de faibles effectifs de chenilles ont consommé le feuillage nouveau, puis, partiellement le feuillage ancien. Lors de ces 2 cycles (1977-1978 et 1978-1979), les effets des 


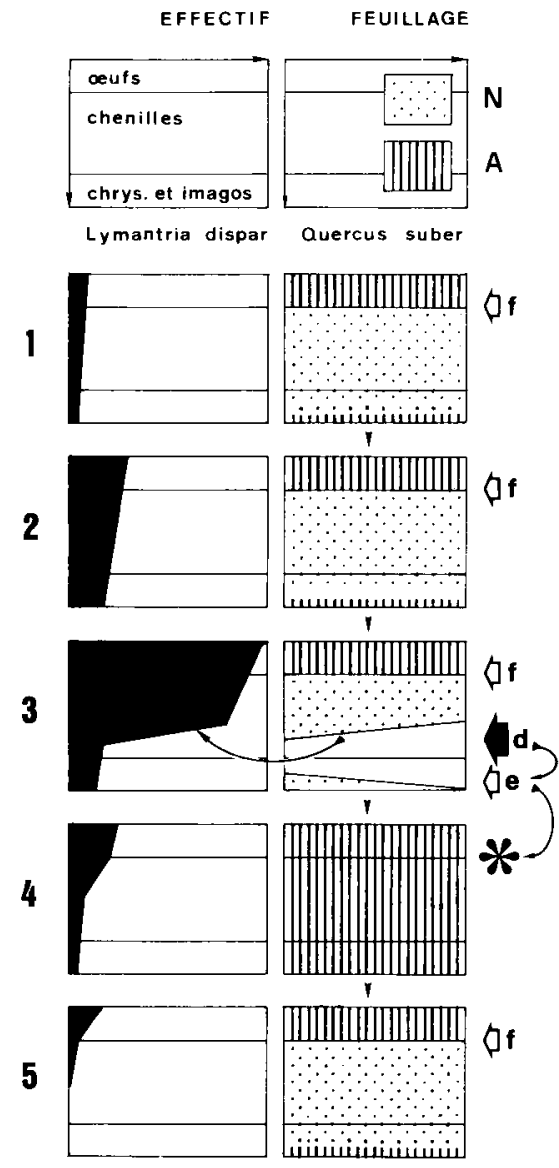

Figure 3

Schéma-type d'une gradation locale de Lymantria dispar (sur 5 années):

- à gauche : évolution des effectifs de $\mathrm{L}$. dispar ; - à droite : évolution du feuillage de Quercus suber; $\mathbf{N}$ : feuillage nouveau, favorable; $\mathbf{A}$ : feuillage ancien, défavorable; $\mathbf{f}$ : débourrement, e : refeuillaison d'été; $\mathbf{d}$ : défoliation ; * discordance phénologique.

Typical 5-year gradation of the gypsy moth:

- right : gypsy moth abundance (eggs, larvae, pupae and adults) - left : cork-oak foliage; $\mathbf{N}$ : young leaves, preferred; $\mathbf{A}:$ old leaves, non-preferred; $\mathbf{f}$ : flushing; $\mathbf{e}:$ summer flushing ; $\mathbf{d}:$ defoliation; * : phenological disruption.

défauts quantitatifs et qualitatifs de la nourriture ont été modérés, permettant le maintien de $L$. dispar en forte densité jusqu'en 1980. A la suite de cette dernière défoliation, les effectifs du ravageur se sont presque annulés en conséquence d'une situation très défavorable, schématisée plus haut ( $c f$. IV A4).

Les effectifs très faibles, voire nuls, constatés avant le printemps 1978 dans une partie de cette zone (stations 118 à 122) sont la conséquence d'un traitement par Bacillus thuringiensis Berl. effectué à cet endroit en 1975 (FRAVAL et al., $1977 a$ et $b$ ) ; la séquence analysée ci-dessus est à mettre en partie au compte de cette intervention (FRAVAL, 1982).

- Une station, en lisière nord-ouest de la forêt, sur une butte exposée aux vents dominants, ne fut pas défeuillée en 1977, contre toute attente, mais seulement en 1978, tardivement, par des chenilles provenant vraisemblablement de l'intérieur de la forêt. FRAVAL \& BENLAHBOUB-JAZOULI (1984) proposent l'explication suivante : la lisière échappe à la défoliation par une émigration des jeunes chenilles qui sont entraînées par le vent ; cette émigration n'étant pas compensée, la population se maintient à un faible niveau, dans de bonnes conditions trophiques car le feuillage est entièrement renouvelé au printemps.

\section{DISCUSSION}

Dans les conditions de la suberaie de la Mamora, les fluctuations des populations de $L$. dispar résultent des variations de la quantité et de la qualité de l'alimentation induites par l'action trophique des chenilles. La privation de nourriture, directement dépendante de la densité, intervient par des effets immédiats (survie, taux sexuel) et différés (fécondité, survie des œufs). La régression est parachevée par un facteur à effet différé, dépendant indirectement de la densité : la nourriture impropre offerte par le chêne à la suite de la défoliation.

Le schéma explicatif des fluctuations numériques locales présenté ici a été bâti dans ses grandes lignes dès 1978, soit au bout de 3 années d'observation (FRAVAL, 1979) ; les 5 années suivantes d'observation n'ont rien apporté qui puisse l'infirmer.

Il importe cependant d'examiner les limites de validité du schéma, lequel est basé sur une autorégulation avec rétroaction négative (due à l'arbre) mécanisme peu fréquent chez les insectes arboricoles, à notre connaissance.

L'intervention d'un tel mécanisme est soupçonnée dans les systèmes $L$. dispar/Quercus velutina Lam. et L. dispar/Betula populifera Marsch par WALLNER \& WALTON (1979). Elle joue un rôle important dans les 2 cas suivants :

- la défoliation de Betula pubescens Ehrh. par la chenille de Epirrita autumnata Bkh. (Lep. Geometridae) induit la résistance de l'arbre au ravageur selon 2 mécanismes (de nature inconnue): l'un, quasi immédiat, s'oppose au développement des individus de la génération en cours, l'autre induit une résistance de longue durée (HAUKIOJA, 1982) ;

- les populations de la tordeuse du mélèze, Zeiraphera diniana Guenée (Lep. Tortricidae) se développent, dans des conditions climatiques favorables, jusqu'à provoquer des modifications du feuillage, lequel devient défavorable, induisant la rétrogradation (BALTENSWEILER et al., 1977).

Parmi les populations de $L$. dispar des suberaies du Maroc atlantique, certaines ne semblent pas gouvernées selon ce mécanisme : dans les forêts de Témara et de Ben Slimane, au sud de Rabat, ou du Gharb et de Larache, plus au nord, $L$. dispar est présent, mais en effectifs toujours très faibles. En forêt de Témara, le débourrement des arbres est presque toujours partiel et tardif, donc du type décrit par CAMUS (1938) ; de plus les arbres sont particulièrement vigoureux ; ces conditions globalement défavorables ne permettent qu'un faible développement des populations de $L$. dispar. Nos renseignements sur les autres forêts sont quasi inexistants.

Par contre, le schéma s'applique très généralement aux évolutions observées (avec une précision bien moindre que sur le transect) dans le canton $\mathrm{A}$, le plus occidental, de la forêt de la Mamora (FRAVAL \& LHALOUI, 1980) ainsi qu'à celles notées (irrégulièrement) 
dans les forêts d'Aïn Felfel (près de Moulay Bou Selham) et de Khemis Sahel (au nord de Larache).

Le schéma ne s'applique qu'à certaines suberaies. Il se pourrait que sa validité soit limitée à un certain stade d'évolution de la forêt. Les conclusions de DE LEPINEY (1930) diffèrent sensiblement des nôtres. Cet auteur considérait notamment que $L$. dispar en forêt de la Mamora pouvait se maintenir plusieurs années de suite en un même lieu en forte densité et attribuait les décalages entre le débourrement du chêne et l'éclosion de $L$. dispar (reconnu comme une cause importante de régression) aux effets du climat.

Des défoliations survenues 5 années de suite d'une même parcelle de la forêt de la Mamora ont été enregistrées (QUESTIENNE, comm. pers.) mais ce phénomène ne s'est pas produit lors de la dernière gradation (1972-1982) où la population s'est effondrée après 2 années de défoliation, au plus. Il est possible que la persistance du feuillage du chêne-liège l'année suivant une défoliation soit liée à l'état actuel de la forêt, notamment à l'âge des sujets : depuis une cinquantaine d'années, les arbres n'ont pratiquement pas été renouvelés, la régénération se faisant par coupes à blanc-étoc.

La dynamique des populations de $L$. dispar en un lieu dépend donc de la phénologie de $Q$. suber, laquelle n'est à ce jour connue, semble-t-il, qu'au travers de nos seules observations, limitées à un seul peuplement et à quelques années.

Dans les limites spatiales et temporelles tracées cidessus, la phénologie de $Q$. suber dépend de la spoliation exercée, l'année précédente, par le défoliateur. D'autres facteurs interviennent, mais leurs actions sont moins nettes et moins régulières. L'âge de l'arbre paraît jouer ( $c f$. IV B) ; des pluies de printemps abondantes, selon une opinion commune des forestiers, provoquent un débourrement complet des arbres dans les forêts où le renouvellement du feuillage est d'ordinaire partiel (ainsi en 1983, en forêt de Témara). Mais l'extraordinaire irrégularité des «comportements» individuels des arbres d'une année sur l'autre n'a pu jusqu'à présent être interprétée. Pour l'étude des relations $L$. dispar/Q. suber à l'échelle de la population nous avons défini une phénologie «moyenne» par station et par date d'observation (FRAVAL et al., 1980) et, dans le présent article, une phénologie « moyenne » par zone et par saison. Ces simplifications, nécessaires pour la représentation du phénomène, masquent le plus souvent une très grande diversité. Toutefois l'observation des chenilles montre que celles-ci ont de grandes chances de vivre sur plusieurs chênes successivement, ces chances et le nombre de sujets augmentant avec la densité et avec l'hétérogénéité phénologique du peuplement; les déplacements intra-arbres et inter-arbres des chenilles ont été peu étudiés (EL YoUSFI, 1980, comm. pers. ; FRAVAL \& BENLAHBOUB-JAZOULl, 1984) ; les effectifs des chenilles ne sont estimés qu'en valeur relative; ainsi nos interprétations demeurent globales.

Nous avons défini la qualité du feuillage offert par un chêne-liège à $L$. dispar par les proportions de feuilles nouvelles et anciennes, descripteur simple mais sans doute insuffisant. En effet, la qualité alimentaire varie certainement d'une feuille à l'autre et d'un arbre à l'autre au sein d'une même catégorie sous l'influence de multiples conditions ( $c f$. II A). Aux effets trophiques de la nature du feuillage sur les populations de $L$. dispar, il conviendrait d'ajouter des effets physiques, par modification du microclimat et par l'offre d'abris, constitués dans la frondaison par les feuilles les plus coriaces. En outre la géométrie de la frondaison (distance existant entre chaque site d'alimentation et celle entre le sol, où la chenille peut tomber, et le feuillage) influe sur la survie des chenilles (DE LÉPINEY, 1930, RAFES \& GINENKO, 1975; HÉRARD, 1984).

Les relations entre $L$. dispar et son arbre-hôte sont certainement plus complexes que ne les décrit notre schéma. En particulier, le synchronisme observé entre les périodes de gradations, en forêt de la Mamora, de Aïn Felfel (100 km au nord) et de Khemis Sahel (100 km au nord de la précédente) et, d'autre part, la mise en évidence d'une relation entre les périodes de gradations enregistrées en forêt de la Mamora depuis 1924 et les variations de l'activité solaire (FARAH, 1980, non publ.), nous suggèrent l'hypothèse d'une action du climat général. Ce dernier définirait des périodes favorables et des périodes défavorables à l'accroissement des populations de $L$. dispar en modifiant la valeur alimentaire des feuilles. La réponse synchrone d'arbres de forêts éloignées serait à rapporter à l'origine commune ancienne (quelques milliers d'années) de ces massifs.

A l'appui de cette hypothèse, on mentionnera le fait que les autres insectes phyllophages de $Q$. suber, compétiteurs directs de $L$. dispar, étaient plus denses pendant la gradation de ce dernier (bien que réprimés par lui) que pendant la phase de latence actuelle, et ceci indépendamment, semble-t-il, de l'intensité et de la fréquence des défoliations en chaque lieu.

Du rôle du facteur trophique, nous ne connaissons que les aspects les plus évidents et les effets sur la réduction des populations de $L$. dispar.

Les aléas climatiques et les ennemis naturels ont été a priori, jugés d'importance mineure, sur la foi de nos $1^{\text {res }}$ observations.

Les effets (hypothétiques) de variations cycliques du climat général viennent d'être évoqués. Parmi les facteurs climatiques, le vent joue un rôle complexe. Nous avons mis en évidence son action en lisière (FRAVAL \& BENLAHBOUB-JAZOULI, 1984). Les courants aériens, comme l'indiquent RABASSE \& BABAULT (1975), sont peut-être responsables de migrations décisives de chenilles pour l'extension des infestations à partir de « foyers ». En forêt de la Mamora, des invasions de zones jusque-là indemnes ont été signalées par divers rapports des agents des Eaux et Forêts (FrAVAL \& LHALOUI, 1980) sans jamais faire l'objet d'investigations précises. L'étude des conditions et de l'amplitude des déplacements aériens des chenilles en suberaie a été seulement ébauchée (EL YOUSFI, 1980, non publ.). Aux U.S.A. le vent est donné pour responsable de l'extension des zones infestées (ANDERSON \& WESELOH, 1981), mais l'efficacité réelle de ce vecteur et son rôle exact restent mal connus (McMANUS \& MASON, 1983). Les pluies de printemps inhibent l'activité des chenilles. Le chergui (vent chaud et sec) a le même effet ; des mortalités de chrysalides lui ont été imputées (EL YOUSFI, comm. pers.).

Pour DE LEPINEY (1930) au Maroc, comme pour 
ROMANYK (1973) en Espagne, CABRAL (1977) au Portugal, RABASSE \& BABAULT (1975) en Tunisie, les ennemis naturels jouent un rôle dépressif important. Les situations que nous avons observées apparaissent différentes : nous n'avons jamais observé d'épizootie et les cas de mortalité importante des chenilles ou chrysalides par l'action d'insectes entomophages ont été rares (FRAVAL \& HÉRARD, 1975) ; le calosome (Calosoma sycophanta L., Col., Carabidae) semble capable, en Mamora occidentale, de faire disparaître localement $L$. dispar (FRAVAL et al., 1981; EL YOUSFI, comm. pers.). Cependant, les relevés opérés sur le transect montrent que l'action des ennemis naturels demeure faible (CHAKIR \& FRAVAL, 1984). Les entomophages semblent jouer un rôle relativement important dans les populations très peu denses mais stables de la forêt de Temara où $L$. dispar est latent. Nous attribuons la faiblesse des ennemis naturels à plusieurs causes : citons, en premier lieu, leur faible diversité, avec l'absence remarquable d'un agent pathogène généralement très efficace, le Baculovirus de $L$. dispar; nous l'avons introduit (Fraval et al., 1981) mais ignorons son devenir, l'hôte étant actuellement extrêmement rare. L'absence de parasites oophages a été palliée par l'introduction d'Ooencyrtus kuvanae How. (Hym. : Encyrtidae) par DE LÉPINEY (1930) ; si cette acclimation est une réussite, son effet sur les populations de $L$. dispar n'est pas perceptible (BENAZOUN \& Fraval, 1983). En second lieu, nous invoquerons la brutalité des gradations de $L$. dispar et leur fréquence relativement faible en un lieu donné qui s'oppose à un développement suffisant des populations d'entomophages ; s'y ajoute la rareté des hôtes de substitution possible, des compétiteurs de $L$. dispar, surtout en période de latence générale de ce dernier.

\section{CONCLUSION}

L'examen régulier, pendant 8 années, d'un échantillon de 565 arbres, répartis sur $7 \mathrm{~km}$ de long dans la forêt de la Mamora a permis de mettre en évidence un mécanisme simple de régulation des populations de $L$. dispar, basé sur 2 caractéristiques du chêne-liège. L'une est spécifique et même générique : l'arbre supporte très bien la défoliation et se refeuille très vite. L'autre semble particulière à certaines forêts du Maroc atlantique, voire à leurs caractéristiques actuel- les : la frondaison supplémentaire persiste régulièrement au printemps suivant.

Cette autorégulation n'est très probablement qu'un élément d'un système plus complexe, qui demeure hypothétique. Elle explique le déclin local d'une population. Les conditions de la progradation restent inconnues; nous ne les avons jamais observées.

Dans son état actuel, le schéma explicatif présente bien des lacunes et ses applications pratiques, pour la maîtrise de la lutte contre le ravageur, sont limitées. Ce schéma a été utilisé, avec succès, pour un programme de surveillance du canton A de la forêt de la Mamora (Fraval \& Lhaloui, 1980). Nous pouvons prévoir qu'un traitement phytosanitaire unique, incomplètement efficace ou appliqué sur une aire restreinte, en période de gradations, se traduira par un report de la pullulation de 2 ou 3 ans. On préconisera en conséquence de lui substituer une défoliation artificielle des arbres (avec un moyen qui reste à définir) avant que des chenilles n'y parviennent. Cette intervention provoquera un effondrement immédiat de la population du ravageur et ménagera des conditions défavorables, l'année suivante, à d'éventuels survivants ou envahisseurs (FRAVAL, 1982).

Les recherches nécessaires pour affiner le schéma, tester les hypothèses formulées, compléter notre compréhension de la dynamique des populations de $L$. dispar sont conditionnées par l'apparition d'une nouvelle période de gradations, dont nous n'avons pas décelé de signe précurseur en 1983. Leur intérêt sera très amoindri si, par ailleurs, le vieillissement non compensé par des régénérations et le surpâturage continuent d'accroître le déclin de la forêt de la Mamora.

Reçu le 2 mars 1983. Accepté le 10 mai 1984.

\section{REMERCIEMENTS}

A F. Hérard, M. Jarry et P. Questienne, nos collaborateurs au début de nos travaux sur $L$. dispar; à J.-P. Dr-PiÉTro et M. EL YOUSFI.

Aux étudiants qui ont apporté leur contribution à ces travaux au terme de leur formation de technicien ou d'ingénieur, tout particulièrement à S. CHAKIR, S. SAIDI et EL M. ZAIMI.

Au personnel technique du Département de Zoologie.

A P. Grison, C. Geri et P. Dumerle qui ont bien voulu relire notre manuscrit.

\section{RÉFÉRENCES BIBLIOGRAPHIQUES}

Anderson J. F., Weseloh R. M., 1981. The gypsy moth in Connecticut : 1 . Defoliation 1975-1980; 2 . Review of biological control studies. The Conn. Agric. Exp. Sta. Newhaven Bull., 797, 25 p.

Baltensweiler W., Benz G., Bovey P., Delucchi V., 1977. Dynamics of larch bud moth populations. Annu. Rev. Entomol., 22, 79-100.

Barbosa P., 1978. Distribution of an endemic larval gypsy moth population among various tree species. Environ. Entomol., 7 (4), 526-527.

Benazoun A., Fraval A., 1983. Etude au laboratoire des performances démographiques d'Ooencyrtus kuvanae. Actes Inst. Agro. Vét. (Maroc), 3, 5-9.
Bess H. A., Spurr S. H., Littlefield E. W., 1947. Forest site conditions and the gypsy moth. Harvard Forest Bull., 22, 56 p.

Boudy P., 1950. Economie forestière nord-africaine t. II, Larose Paris.

Cabral M. T., 1977. Papel das doenças ne limitaçao natural das populaçoes de Lymantria dispar L. (Lepidoptera Lymantriidae). An. Inst. Super. Agron. Lisboa, 37, 153-177.

Campbell R. W., Sloan R. J., 1978. Numerical bimodality among North American gypsy moth populations. Environ. Entomol., 7 (5), 641-646. 
Camus A., 1936-1938. Les chênes. Encyclopédie Economique de sylviculture, t. VI. Paul Lechevalier, Paris.

Chakir S., Fraval A., 1984. Les ennemis naturels et introduits de Lymantria dispar (L.) (Lep. : Lymantriidae) en forêt de la Mamora (Maroc) ; Etude le long d'un transect : 1976-1982. Actes Inst. Agro. Vét. (Maroc), 4, (sous presse).

De Lépiney J., 1930. Contribution à l'étude du complexe biologique de Lymantria dispar L. Mém. Soc. Sci. Nat. Maroc, 23, 100 p.

Doane C. C., 1968. Changes in egg-mass density, size and amount of parasitism after chemical treatment of a heavy population of the gypsy moth. J. Econ. Entomol., 61 (5), 1288-1291.

Feeny P., 1970. Seasonal changes in oak tannins and nutrients as a cause of spring feeding by winter moth caterpillars. Ecology, 51, 565-581.

Forbush E. H., Fernald C. H., 1896. The gypsy moth : Porthetria dispar Linn. Part I. Wright \& Potter Printings Co., Boston. $251 \mathrm{p}$. In R. W. Campbell, L. C. Levitan, E. R. Sobecki, M. F. Tardif. Population dynamic of the gypsy moth: an annotated bibliography. Forest service general technical report NE-48. Forest Service U.S.D.A. Northeastern forest expt. Sta. 125 p.

Fraval A., 1979. Effets démécologiques du facteur trophique sur Lymantria dispar en suberaie marocaine atlantique. In A. Fraval, P. Questienne \& J. P. Di-Piétro : Compte rendu du séminaire tenu à Rabat du 5 au 7 février 1979 sur Lymantria dispar. Doc. Labo. de Zoologie. I.A.V. HASSAN II 100 p. + ill.

Fraval A., 1982. Méthodes de lutte contre Lymantria dispar en suberaie marocaine. Actes Inst. Agro. Vét. (Maroc), 2, 92-117.

Fraval A., 1983. Echantillonnage de Lymantria dispar en suberaie marocaine atlantique. In S. Frontier: Stratégies d'échantillonnage en écologie animale. Masson, Paris, 273-290.

Fraval A., Benlahboub-Jazouli H., 1984. Etude de Lymantria dispar L. (Lep. : Lymantriidae) en lisière de suberaie ; forêt de la Mamora, Maroc. Actes Inst. Agro. Vét. (Maroc), 4 (1), (sous presse).

Fraval A., Hérard F., 1975. Programme de prospecilon de la faune antagoniste de Lymantria dispar L. au Maroc. Rapport final. USDA. Grant FG-Mo-102; Projet $n^{\circ}$ F12-ENT-1. Doc. Labo. de Zoologie, I.A.V. HASSAN II, 45 p. + ill.

Fraval A., Lhaloui S., 1980. La surveillance des attaques de Lymantria dispar L. en forêt de Mamora (canton A) : 1980. Ann. Rech. For. Maroc, 20, 351-386.

Fraval A., Mazih A., 1980. Mortalité de Lymantria dispar L. (Lep. Lymantriidae) sur trois placeaux de la suberaie de la Mamora, en 1978. Ann. Rech. For Maroc, 20, 288-296.

Fraval A., Hérard F., Jarry M., 1978. Méthodes d'échantillonnage des populations de pontes de Lymantria dispar (Lep. : Lymantrildae) en Mamora (Maroc). Ann. Zool. Ecol. Anim., 10 (2), 267-279.

Fraval A., Hérard F., Questienne P., 1975. Connaissances actuelles sur la situation au Maroc d'un ravageur mondial des forêts : Lymantria dispar L., Hommes, Terre, Eaux, 4 (17), 47-53.

Fraval A., Jarry M., Questienne P., 1977a. Traitements expérimentaux contre Lymantria dispar (Lep. Lymantriidae) en suberaie de la Mamora au moyen de diflubenzuron et de deux préparations à base de Bacillus thuringiensis Berl., Ann. Rech. For. Maroc, 17, 77-92.

Fraval A., Questienne P., Jarry M., 1980. Démographie de Lymantria dispar L. en suberaie de la Mamora ; étude le long d'un transect ; 1976-1977-1978. Ann. Rech. For. Maroc, 20, 3-64.

Fraval A., Hérard F., Jarry M., Questienne P., Viland M. Cl., Vein D., $1977 a$. Note sur un traitement au moyen d'une préparation de
Bacillus thuringiensis Berliner contre Porthetria dispar $\mathrm{L}$. en forêt de la Mamora (Maroc). Ann. Rech. For. Maroc, 17, 67-76.

Fraval A., Mazih A., El Yousfi M., Saidi S., Chakir S., 1981. Introduction du Baculovirus de Lymantria dispar $\mathbf{L}$. en forêt de la Mamora (Maroc). Ann. Rech. For. Maroc, 21, 297-315.

Goldschmidt R., 1934. Lymantria. Bibliogr. Genet., 11, 1-186.

Haukioja E., 1982. Inducible defences of white birch to a geometrid defoliator, Epirrita autumnata. Proc. Sth. Int. Symp. Insect Plant Relationships, Wageningen, Pudoc, 199-203.

Hérard F., 1984. Etude des relations trophiques entre Lymantria dispar L. (Lep. Lymantriidae) et Quercus suber L. (Fagacae) dans les conditions - simulées en laboratoire - de la forêt de Mamora (Maroc) : 1. Enoncé général des résultats. Actes Inst. Agro. Vét (Maroc), 4 (1), (sous presse).

Hérard F., Fraval A., 1980. La répartition et les ennemis naturels de Lymantria dispar L. (Lep. Lymantriidae) au Maroc, 1973-1975. Acta OEcol. OEcol. Appl., 1 (1), 35-48.

Hough J. A., Pimentel D., 1978. Influence of host foliage on development, survival and fecundity of the gypsy moth. Environ. Entomol., 7 (1), 97-102.

Leonard D. E., 1966. Effects of starvation on behaviour, number of larval instars and developmental rate of Porthetria dispar. J. Insect Physiol., 16, 25-31

Leonard D. E., 1970. Intrinsic factors causing qualitative changes in populations of Porthetria dispar (Lepidoptera, Lymantriidae). Can. Entomol., 102, 239-249.

McManus M. L., Mason C. J., 1983. Determination of the settling velocity and its significance to larval dispersal of the gypsy moth (Lepidoptera, Lymantriidae). Environ. Entomol., 12, 270-272.

Patočka J., 1973. Einfluss der Nahrung und des Standortes auf die Mortalität einiger an Fichten lebenden schädlichen Lepidopteren. Vestn Cesk. Spol. Zool., 37 (4), 282-292.

Potts S. F., 1938. The weight of foliage from different crown levels of trees and its relation to insect control. J. Econ. Entomol., 31 (5), $631-632$.

Rabasse J. M., Babault M., 1975. Etude d'une pullulation de Lymantria dispar L. (Lep. Lymantriidae) dans les conditions méditerranéennes. Sci. Agron., 143-159.

Rafes P. M., Ginenko Y. I., 1973. The survival of leaf eating caterpillars (Lepidoptera) as related to their behaviour. Entomol. Rev., 52, 204-211.

Ridet J. M., 1972. Etude des conditions optimales d'élevage et d'alimentation de Lymantria dispar L., Ann. Soc. Entomol. Fr., (N.S.), 8 (3), 653-668.

Romanyk M., 1973. Les gradations de Lymantria dispar L. en Espagne. Zast. bilja, 124-125, 285-288.

Schedl K. E., 1936. Der Schwammspinner (Porthetria dispar L.) in Euroasien, Afrika und Neuengland. Z. angew. Entomol. Monogr., 12, $242 \mathrm{p}$.

Schindler M., 1921. Lymantria dispar en forêt de la Mamora. Bull. Soc. Sci. Nat. Maroc., 1, 144-145.

Von Rudnev D. E., 1963. Physiologischer Zustand der Wirtspflanze und Massenvermehrung von Fortschädlingen. Z. Angew. Entomol., 53, $48-68$.

Wallner W. E., Walton G. S., 1979. Host defoliation : a possible determinant of gypsy moth population quality. Ann. Entomol. Soc. Am. 72, 62-67. 